\title{
\begin{tabular}{l|} 
EXPERT \\
REVIEWS
\end{tabular}
}

\section{TORAFIC study protocol: torasemide prolonged release versus furosemide in patients with chronic heart failure}

Expert Rev. Cardiovasc. Ther. 7(8), 897-904 (2009)

Javier Díez ${ }^{\dagger}$, Antonio Coca, Eduardo de Teresa, Manuel Anguita, Alfonso Castro-Beiras, Pedro Conthe, Erik Cobo, Ester Fernández; on behalf of the TORAFIC Investigators Group ${ }^{+}$Author for correspondence Division of Cardiovascular Sciences, Centre of Applied Medical Research, and Department of Cardiology and Cardiovascular Surgery, University Clinic, Universidad de Navarra, Edificio CIMA, Avda. Pío XII 55, E-31008,

Pamplona, Spain

Tel.: +34 9481947000

Fax: +34 948194716 jadimar@unav.es

Loop diuretics, such as torasemide and furosemide, are important agents in the treatment of chronic heart failure. Beneficial effects of torasemide immediate-release formulation beyond diuresis have been documented as the ability of this compound to inhibit myocardial synthesis and deposition of collagen type I in patients with chronic heart failure. In addition, torasemide-treated patients, but not furosemide-treated patients, showed decreased serum concentrations of the C-terminal propeptide of procollagen type I, a biochemical marker of myocardial fibrosis. The aim of the TORAFIC study is to test the efficacy of torasemide prolonged-release formulation (PR) in reducing myocardial fibrosis in chronic heart failure in a large, randomized clinical trial. Methods: This prospective, Phase IV, randomized, blinded end point, active-controlled clinical trial will randomize 142 patients with chronic heart failure in New York Heart Association functional class II-IV to 8 months treatment with either torasemide-PR (10-40 mg daily) or furosemide (40-160 mg daily). The primary objective is to test the hypothesis that torasemide-PR is superior to furosemide in reducing myocardial fibrosis. The primary outcome measure is the difference in the change of serum propeptide of procollagen type I concentration from the initial to the final visit between both study groups. Secondary outcome measures include all efficacy variables related to heart failure (signs and symptoms, ECG, echocardiogram and serum levels of $\mathrm{N}$-terminal brain natriuretic propeptide). Secondary safety variables are heart rate, blood pressure, laboratory data, adverse events, cardiovascular events (hospital admission, emergency department visits) and quality of life (Minnesota questionnaire). Discussion: This trial will test whether torasemidePR possesses antifibrotic properties, which may provide an additional benefit beyond diuresis in patients with chronic heart failure.

KEYWORDS: chronic heart failure $\bullet$ furosemide $\bullet$ myocardial fibrosis $\bullet$ torasemide

The prevalence of symptomatic heart failure has been estimated to be between 0.4 and $2 \%$ in the European general population [1]. A recent study carried out in Spain showed that the prevalence of heart failure increases with age in people over 45 years, being $16.1 \%$ in those aged 55-64 years [2]. The epidemiological profile of heart failure in Western society has a considerable social impact due to the increasing prevalence of the disease in relation to aging of the population and increased life expectancy [3]. In addition, because heart failure has a poor prognosis [4] with a 4-year mortality rate of $50 \%$, there is an urgent need to establish an adequate diagnosis in order to treat patients promptly and to implement more efficient therapeutic strategies to minimize economic burden on health services due to the cost of long-term treatment and frequent hospitalization [5].

Various conceptual pathophysiological models have been used for envisioning the syndrome of heart failure, including excessive salt and water retention, reduced cardiac output and excessive peripheral vasoconstriction, and deleterious changes in cardiac function and 
structure as the result of sustained neurohormonal activation [6]. The underlying cellular and molecular basis of myocardial structural remodeling, an important contributory event in the progression of heart failure, has been investigated extensively. It has been proposed that the excess of myocardial collagen seen in the failing heart is primarily a result of the uncoupling between increased formation and unchanged or decreased degradation of collagen type I fibers, in which hemodynamic loading, ischemia, hormones and growth factors may be involved [7,8]. Myocardial fibrosis is now considered a major determinant of altered diastolic filling and compromised systolic pump function $[9,10]$. In particular, available clinical and experimental evidence suggests that an excessive myocardial collagen type I synthesis and deposition is involved in the development of heart failure in hypertensive heart disease [11].

Emerging experimental and clinical experience holds promise for the determination of various serum peptides derived from the metabolism of collagen type I as a noninvasive way to assess myocardial fibrosis in heart diseases. More specifically, the serum concentration of the $\mathrm{C}$-terminal propeptide of procollagen type I or procollagen type I (PICP), a peptide that is cleaved from procollagen type I during the extracellular synthesis of fibril-forming collagen type I and that is released into the blood stream with a stoichiometric ratio of $1: 1$, has been shown to be associated with the volume of myocardial tissue occupied by collagen fibers (or collagen volume fraction) in hypertensive patients with [12] and without [13] heart failure. It has also been shown that the variation in serum PICP concentration induced by treatment is associated with parallel changes in the amount of myocardial collagen type I fibers in treated hypertensive patients [14]. PICP measurable in serum is mostly of cardiac origin, as a positive gradient from coronary sinus blood concentrations to peripheral vein blood concentrations has been reported [13] and, thus, is now considered a reliable biochemical marker of myocardial fibrosis [15].

Loop diuretics, such as torasemide and furosemide are currently recommended by the European Society of Cardiology [16], the American College of Cardiology and the American Heart Association guidelines on treatment of heart failure [17]. The improvement in left ventricular function has been reported to be superior in heart failure patients receiving torasemide immediate release (IR) as diuretic treatment than in patients receiving furosemide [18]. Although not designed as a mortality study, data from a previous, large, open-label, cohort trial suggested a lower mortality among chronic heart failure patients who were treated with torasemide compared with furosemide/other diuretics [19].

The pharmacological properties, clinical efficacy and safety profile of torasemide-IR are well described [20,21]. It has been shown that bioavailability, pharmacokinetics and pharmacodynamics of torasemide in patients with congestive heart failure are comparable to those in healthy volunteers $[22,23]$. In addition, torasemide-IR has a longer half-life and duration of action, and higher bioavailability compared with furosemide [23]. A new, recently developed torasemide prolonged-release (PR) formulation has shown lower peak plasma levels while maintaining equivalent AUC of plasmatic levels compared with the IR formulation [24-26]. Moreover, a recent randomized noninferiority trial demonstrated that both PR and IR torasemide formulations were well tolerated and showed similar efficacy, tolerability and safety profiles in a population of mild-to-moderate hypertensive patients [27].

\section{Rationale for study}

The currently available data suggest that torasemide-IR may be a better alternative than furosemide for the treatment of congestive heart failure. In part, this may be due to the fact that, in contrast to furosemide, torasemide-IR appears to have beneficial pharmacokinetic properties and pharmacodynamic actions, even in patients with congestive heart failure. Torasemide also appears to have additional actions beyond the pure loop diuretic effect, such as anti-aldosterone and vasorelaxant actions [18]. Torasemide has also been demonstrated to have antifibrotic effects in the heart. In fact, studies in rats $[28,29]$ and humans [30-32] with heart failure have demonstrated that, whereas treatment with torasemide-IR was associated with a reduction in the amount of histologically proven myocardial fibrosis (as assessed by measuring the collagen volume fraction), treatment with furosemide did not. Interestingly, while serum concentration of PICP was reduced after torasemideIR treatment, it remained unchanged in furosemide-treated patients [30,31]. In addition, a direct correlation was found between changes in serum PICP and changes in collagen volume fraction in torasemide-IR-treated patients [30,31]. Molecular data show that torasemide-IR interferes with the action of the myocardial enzyme procollagen type I C-terminal proteinase, which forms collagen type I molecules and the myocardial enzyme lysyl oxidase, which, in turn, processes these molecules to form the final collagen type I fibers $[31,32]$.

Whether the ability of torasemide-IR to reduce cardiac synthesis and deposition of collagen type I fibers in heart failure patients is also shared by torasemide-PR is still unknown.

\section{Methods/design \\ Study objectives}

The primary objective of this clinical trial is to determine the possible superiority of torasemide-PR compared with furosemide in reducing the serum concentration of PICP, a biochemical marker of myocardial fibrosis in patients with chronic heart failure in New York Heart Association (NYHA) functional class II, III and IV.

Secondary objectives include the comparison of torasemide-PR versus furosemide in relation to changes in signs and symptoms of chronic heart failure, including edemas (measurement of body weight), NYHA functional class and urinary urgency; clinical parameters (blood pressure, heart rate and renal function); hospitalizations and/or extra visits and/or emergency department consultations $(<24 \mathrm{~h})$ owing to cardiovascular manifestations related to heart failure, safety and tolerability; and changes in quality of life [33]. 


\section{Study design}

This is a prospective, Phase IV, randomized, blinded end point, active-controlled drug clinical trial (prospective randomized open blinded end-point [PROBE] design; Figure 1) [34]. The study is being carried out in 20 acute-care tertiary hospitals and seven primary care centers in Spain. A total of 142 subjects will be included in the study. The duration of the study is 8 months. Approval was obtained from the National Health Authorities and institutional review boards of the participating hospitals (Reference Ethics Committee, Hospital Clínic i Provincial, Barcelona, Spain). All study participants need to sign an informed consent.

\section{Study population}

Potential study subjects are those with chronic heart failure as defined by the European Society of Cardiology [16], with either reduced or preserved left ventricular ejection fraction (left ventricular ejection fraction $>50 \%$ ) in accordance with criteria of the Heart Failure and Echocardiography Associations of the European Society of Cardiology [35], with a history of arterial hypertension according to categories defined by the European Society of Hypertension/European Society of Cardiology Guidelines Committee [36], and who require loop diuretic therapy for maintaining euvolemia. Subjects are identified from hospitalized patients or patients attending the outpatient clinics of departments of cardiology or hypertension units in the acute-care tertiary hospital setting and primary care centers.

\section{Inclusion/exclusion criteria}

The selection criteria used in the study are shown in Box 1.

\section{Overall study description}

A detailed description of the different steps of the study is shown in Table 1. Consecutive patients who agree to participate will be randomized to treatment with torasemide-PR or furosemide. Both medications are dispensed in their usual different pharmaceutical forms, allowing both physician and patient to identify their concrete active treatment. At 7 days, a visit will be scheduled to collect daily cards (recording of urinary urgency). Patients will be seen 4 weeks after initiation of active treatment and in those who do not respond to treatment with torasemide-PR $10 \mathrm{mg} /$ day or furosemide $40 \mathrm{mg} /$ day, the dose will be increased to 20 and $80 \mathrm{mg} /$ day, respectively. Patients responding to treatment with torasemide-PR $10 \mathrm{mg} /$ day or furosemide $40 \mathrm{mg} /$ day will continue with this dose until the following visit. They will be seen again 12 and 24 weeks after the start of treatment and those who do not respond to the initial doses will receive torasemide-PR $20 \mathrm{mg} /$ day or furosemide $80 \mathrm{mg} / \mathrm{day}$. Nonresponders to torasemide-PR $20 \mathrm{mg} /$ day may have a $50 \%$ dose increase (30 mg/day) or $100 \%$ dose increase ( $40 \mathrm{mg} /$ day) at the discretion of the investigator. Nonresponders to furosemide $80 \mathrm{mg} /$ day may have a $50 \%$ dose increase $(120 \mathrm{mg} /$ day $)$ or $100 \%$ dose increase $(160 \mathrm{mg} /$ day $)$ at the discretion of the investigator. Patients who do not respond with torasemide-PR $40 \mathrm{mg} /$ day or furosemide $160 \mathrm{mg} /$ day will be withdrawn from the study medication and followed until the final visit. The last visit will take place 32 weeks after the treatment initiation. The total duration of treatment with torasemide-PR or furosemide will be 8 months. The treatment schedules of torasemide-PR and furosemide are shown in Table 1.

Prohibited medications include any diuretic other than the trial medication, NSAIDs in a treatment of over 7 consecutive days (including salicylates), concomitant treatment with lithium, probenecid, aminoglycoside antibiotics, etacrynic acid, nasal vasoconstrictive drugs, systemic corticosteroids, monoamine oxidase inhibitors and all investigational drugs.

\section{Randomization}

Once consecutive patients fullfilling eligibility criteria consent to be included, the researcher will inform the pharmacy department, who will send the allocated medication following the randomization list previously generated by a central statistician in blocks. It will consist of three digits (from 001 to 180), which will have been previously assigned to each center.

\section{Screening \& follow-up visits}

At the screening visit, the trial will be explained and written informed consent obtained. Medical history, concomitant conditions and medications will be recorded, and a physical examination carried out. Results of clinical laboratory tests, 12-lead ECG, echocardiogram and chest roentgenogram will be reviewed, as well as inclusion/exclusion criteria. Adverse events arising after signing the informed consent will be documented. The randomization visit (visit 0 ) will be performed 7-10 days after the screening. The following procedures will be performed:

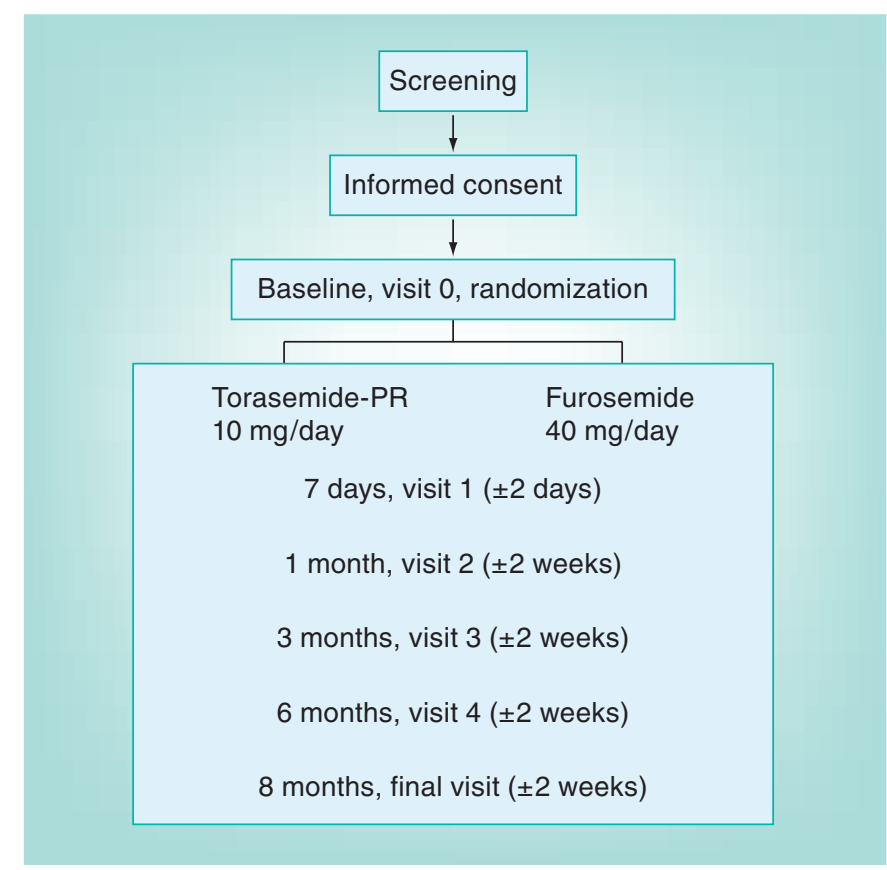

Figure 1. Prospective, Randomized, Open, Blinded End point (PROBE) study design.

PR: Prolonged release. 


\section{Box 1. Inclusion and exclusion criteria for the TORAFIC study.}

\section{Inclusion criteria}

- Men or women aged over 18 years

- Patients with chronic heart failure as defined by the European Society of Cardiology (update 2005) [19] with reduced or preserved left ventricular ejection fraction (>40\%), class I-IV (New York Heart Association) due to arterial hypertension diagnosed according to categories defined by the 2003 European Society of Hypertension

- Subjects who required diuretic therapy for maintaining euvolemia during the previous 3 months

- Patients with left ventricular hypertrophy diagnosed by echocardiogram

- Patients without ischemic cardiopathy or nonrecent ischemic cardiopathy (acute myocardial infarction for more than 6 months prior to the study, or coronary syndrome, acute cerebrovascular accident or peripheral vascular disease for more than 3 months prior to the study)

- Capable of understanding the nature of the trial

- Signed informed consent

\section{Exclusion criteria}

- Heart failure due to aortic stenosis or hypertrophic cardiomyopathy

- Recent coronary syndrome, acute cerebrovascular event or peripheral vascular disease (less than 3 months)

- Recent myocardial infarction (less than 6 months)

- Unstable angina pectoris

- Severe cardiac arrhythmia (sustained ventricular tachycardia, atrial fibrillation with accompanying ventricular tachycardia, atrial flutter, bradycardia under 45 bpm)

- Pregnancy or breastfeeding

- Treatment with aldosterone antagonists in the previous 6 months

- Known hypersensitivity to the study drugs or sulphonylureas

- Liver disease (alanine aminotransferase or aspartate aminotransferase over twice upper limit of normal)

- Chronic renal failure defined by the following parameters: serum creatinine $>2.5 \mathrm{mg} / \mathrm{dl}$ and/or glomerular filtration rate $<30 \%$

- Noncontrolled insulin-dependent diabetes

- Contraindications in the data obtained during the selection process in the physical examination, hematology, biochemistry, urinalysis and 12-lead electrocardiogram

- Patients included in another simultaneous study or receiving treatment with any investigational drug within 30 days prior to signing informed consent

- Lactose intolerance

- Concomitant lithium treatment

- Chronic treatment (>7 days) with NSAIDs, including aspirin

- Concomitant treatment with aminoglycoside antibiotics, etacrynic acid

- History of drug or alcohol dependence within the 6 months prior to the start of the trial

- Current loop diuretic treatment over study doses (torasemide $>10 \mathrm{mg} /$ day, furosemide $>40 \mathrm{mg} /$ day) or patients requiring higher doses within 30 days prior to the study

- Any condition that, in the investigator's opinion, would prevent the safe completion of the study protocol of the administration of torasemide prolonged release or furosemide safely

signs and symptoms of heart failure (presence/absence S3 gallop, hepatojugular reflux, jugular venous distention, dyspnea [at rest and on effort]); assessment of peripheral edema; assessment of NYHA functional class; review of inclusion/exclusion criteria and randomization to one of the trial treatments if the patient complies with the eligibility criteria; a blood sample will be obtained for measurement of serum concentrations of PICP and $\mathrm{N}$-terminal brain natriuretic propeptide (NT-proBNP) by specific ELISA methods; the Minnesota test will be administered; urinary urgency will be recorded (a daily card will be provided to register urinary urgency symptoms on three alternative days during the first 7 days of treatment); medication will be issued for the first month of treatment; and the patient will be given an appointment for 7 days ( \pm 2 days; visit 1$)$. At visit 1 , NYHA functional class, body weight, heart rate and blood pressure will be recorded, the completed daily card will be collected, and the patient will be given an appointment for 4 weeks' time ( \pm 5 days; visit 2 ). At visit 2 , assessments will include: NYHA functional class, body weight, heart rate, blood pressure, ionogram, serum creatinine and glomerular filtration rate, signs and symptoms of heart failure, peripheral edema, urinary urgency, concomitant medication, adverse events and response to treatment. Medication will be issued for the second month of treatment and the patient will be given an appointment for 


\section{Table 1. Treatment scheme in the torasemide prolonged release and furosemide (active comparator) arms.}

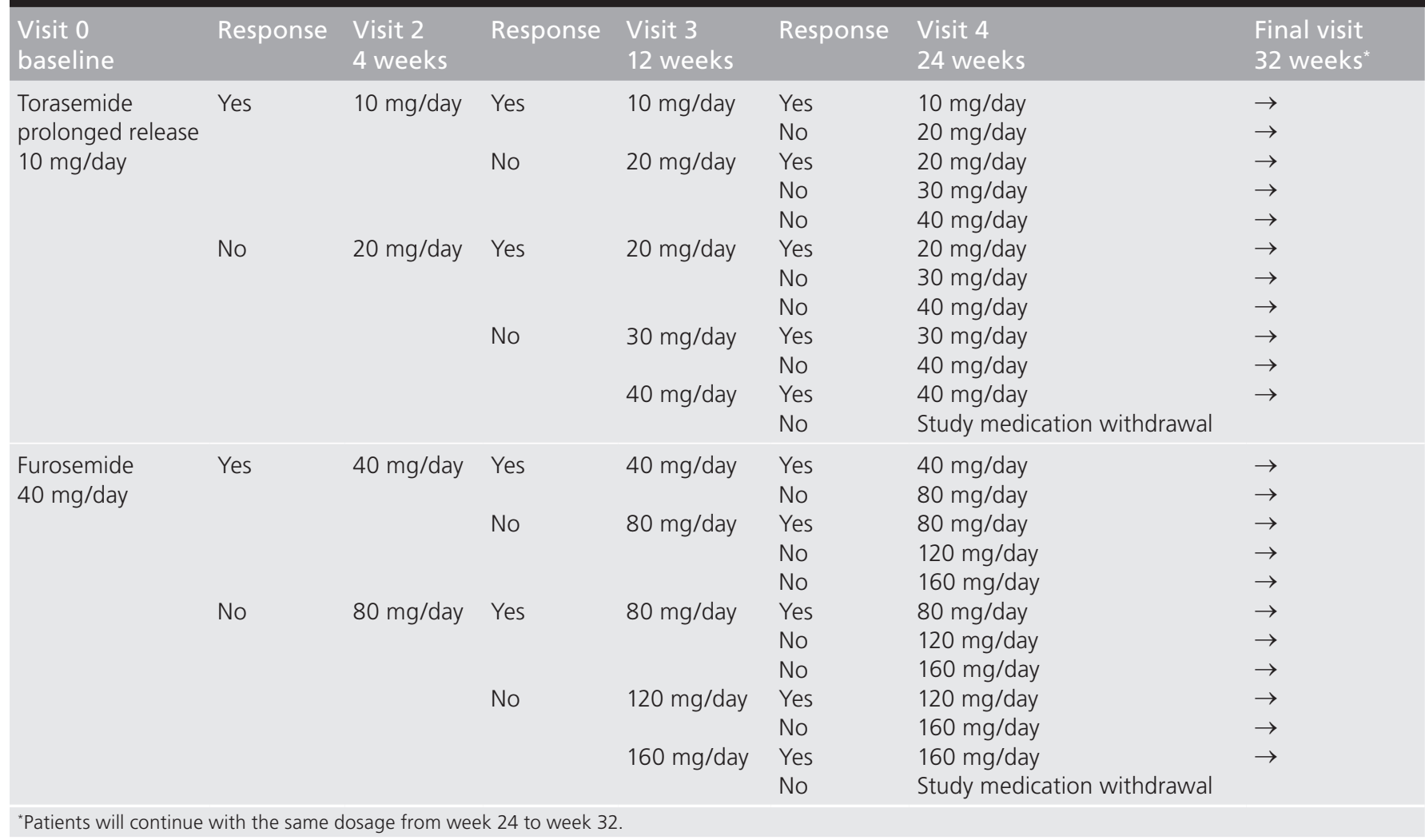

12 weeks' time ( \pm 2 weeks; visit 3 ). Visit 3 includes the same procedures as visit 2 . In addition, a blood sample will be taken for complete blood count, ionogram, biochemical profile, serum creatinine and glomerular filtration rate, and 12-lead ECG will be performed. Medication will be issued for the fourth, fifth, and sixth month of treatment and the patient will be given an appointment for 12 weeks' time ( \pm 2 weeks; visit 4$)$. At visit 4 , the same procedures as those described for visit 2 will be performed. Medication will be issued for the seventh and eighth month of treatment and the patient will be given an appointment for 8 weeks' time ( \pm 2 weeks; visit 5 , final visit). Visit 5 (follow-up visit after $32 \pm 2$ weeks of treatment) includes all procedures performed in visit 3 together with echocardiogram, chest roentgenogram and the Minnesota test, assessment of urinary urgency, and measurement of PICP and NT-proBNP. At each appointment the patient will be told to attend on an empty stomach without taking the trial medication and bring any unused medication.

At each follow-up visit, the investigator will ask the patient about the presence of urinary symptoms, including the urgent need to urinate, its intensity (none, mild, intense and unbearable) and frequency, and the presence of nocturia and its frequency.

If one randomized patient drops out before 3 months of follow-up, the examinations corresponding to visit 3 will be performed and the corresponding information recorded. If one randomized patient drops out after 3 months of follow-up, the examinations corresponding to visit 5 will be performed and the corresponding information recorded. Owing to the dynamics of collagen type I metabolism, PICP will be determined only in patients who do not drop out before 24 weeks of follow-up.

\section{Nonresponse criteria}

If improvement in circulatory congestion (as assessed by clinical and/or radiological disappearance of signs of pulmonary congestion and weight loss $>2 \mathrm{~kg}$ ) owing to fluid retention compared with baseline or the previous follow-up visit is not observed, the patient will be considered to have not responded to treatment. Heart failure-related clinical manifestations include: body weight, vital signs, and signs and symptom of congestive heart failure: S3 gallop, hepatojugular reflux, jugular venous distention, dyspnea at rest and on effort, peripheral edema, and NYHA functional class.

\section{End points}

\section{Primary end point}

The primary end point is the difference in serum PICP concentrations recorded at the final visit after $32 \pm 2$ weeks of treatment compared with the initial visit. Serum PICP is provided by a centralized laboratory unaware/concealed of allocated treatment.

\section{Secondary end points}

Secondary end points include all secondary efficacy variables related to the clinical course of heart failure, such as body weight, peripheral edema, signs and symptoms of congestive heart failure, ECG recordings, echocardiogram and serum levels of 
NT-proBNP. Secondary safety variables include vital signs (heart rate and blood pressure), laboratory tests (blood count, ionogram, renal function and biochemical profile), quality of life (Minnesota questionnaire) and adverse events.

\section{Safety considerations}

Safety assessments will include monitoring and recording all adverse events and serious adverse events, measurements of vital signs, monitoring of blood biochemistry, ECG recordings and physical examination. The causal relationship of adverse events with the study medication will be assessed according to the Karch and Lasagna classification [37].

\section{Statistical analysis}

\section{Power calculation \& sample size}

Evaluable patients will be those who meet all inclusion criteria and nonexclusion criteria, complete the 8 months of treatment and attend the study visits. The sample size has been calculated based on the primary study variable. In the study by López et al., mean serum concentrations of PICP at the end of the study were $111 \mu \mathrm{g} / \mathrm{l}$ in the torasemide-IR group and $133 \mu \mathrm{g} / \mathrm{l}$ in the furosemide group, with a punctual estimation of the size effect of $22 \mu \mathrm{g} / \mathrm{l}$ (95\% CI: 7-37) [30]. Standard errors in both groups of 19 and 17 cases were 3 and $7 \mu \mathrm{g} / \mathrm{l}$, respectively, with a punctual estimation of the standard error of 22 (95\% CI: 18-29). If a difference between treatment of $15 \mu \mathrm{g} / \mathrm{l}$ and a standard error of 29 (both values included in their 95\% CIs) are considered as clinically relevant, the sample size required at the $\alpha=p<0.05$ (two-sided) and a power (1- $\beta$ ) of $80 \%$ is 59 patients per group (a total of 118). Foreseeing 20\% postrandomization losses, it would be necessary to randomize 142 patients.

The recruitment of patients finished in October 2008, with 169 patients.

\section{Analyses}

Differences in serum concentration of PICP between the study groups (primary objective of the study) will be assessed using an analysis of covariance (ANCOVA) model, with baseline PICP as the covariable. For the comparison of torasemide-PR versus furosemide regarding the variables included in the secondary objectives of the study, parametric and nonparametric statistical tests will be used when appropriate, such as the Student's t test, the Mann-Whitney U test, the chi-square $\left(\chi^{2}\right)$ test or the Fisher's exact test. Principal analysis of the study is the full analysis set or intention-to-treat population for all randomized patients taking at least one dose of the trial medication. Other statistical analyses will include the per-protocol efficacy analysis for all randomized patients who have concluded the trial as established in the protocol, and the safety population for all randomized patients taking at least one dose of the trial medication.

\section{Discussion}

Given the importance of fibrous tissue accumulation in myocardial dysfunction and failure in hypertensive heart disease, noninvasive assessment of fibrosis is a clinically useful tool in patients with heart failure, particularly given the potential for cardioprotective and cardioreparative pharmacological strategies [38]. In this conceptual framework, the measurement of serum concentrations of PICP represents an exciting and innovative approach. As mentioned previously, the available evidence suggests that the goal of reducing myocardial fibrosis is achievable in patients with chronic heart failure using long-term treatment with torasemide-IR [30-32]. This clinical trial will test whether torasemide-PR also possesses antifibrotic properties (as assessed by its ability to reduce serum PICP concentration) that in turn may provide an additional benefit beyond diuresis in patients with chronic congestive heart failure receiving this agent.

\section{Acknowledgements}

All authors are involved in the design and acquisition, analysis and interpretation of data. They have been involved in drafting the manuscript and revising it critically for important intellectual content and have given final approval of the version to be published.

Members of the TORAFIC Investigators Group: Eulalia Roig, Hospital Clinic, Barcelona, Spain; Enrique Galve, Hospital da la Vall d' Hebron, Barcelona, Spain; Josep Lupón, Hospital Germans Trias i Pujol, Badalona, Barcelona, Spain; Francisco Ridocci, Hospital General de Valencia, Valencia, Spain; Domingo Pascual, Hospital Virgen de la Arrixaca, Murcia, Spain; Pedro L Sánchez, Hospital Gregorio Marañón, Madrid, Spain; Cándido Martín, Hospital Universitario de Salamanca, Salamanca, Spain; Juan Ignacio Pérez-Calvo, Hospital Clinico Universitario, Zaragoza, Spain; Ramón Querejeta, Hospital de Donostia, San Sebastián, Spain; Manuel Jiménez-Navarro, Hospital Clínico, Málaga, Spain; Lorenzo Monserrat, Complejo Hospitalario Universitario de La Coruña, La Coruña, Spain; JR González-Juanatey, Hospital Clinico Universitario, Santiago de Compostela, Spain; Beatriz Diaz, Hospital Central de Asturias, Oviedo, Spain; Jesús Cebollada, Hospital San Jorge, Huesca, Spain; Julia Roure, Hospital Josep Trueta, Girona, Spain; Sonia Ruiz, Hospital Universitari del Mar, Barcelona, Spain; Isidro López Centro de Salud Begonte, Lugo, Spain; Manel Terns, CAP Remei, Vic, Barcelona, Spain; Silvia Narejos, CAP Centelles, Barcelona, Spain; Alex Rodríguez, CAP Alcover, Tarragona, Spain; Mar Rodriguez, ABS Canet de mar, Barcelona, Spain; and Pere Toran, EAP Mataró 6 (Gatassa), Barcelona, Spain.

\section{Financial \& competing interests disclosure}

This trial received funding from Laboratorios Novag SA (Ferrer Internacional, Barcelona, Spain). Trial registration is EudraCT 2006001446-14; FDA (Clinicaltrials.gov) NCT00409942. Erik Cobo is a consultant for Ferrer International and Medtronic (UK) and receives regular training fees from both institutions and Novartis (UK). The authors have no other relevant affiliations or financial involvement with any organization or entity with a financial interest in or financial conflict with the subject matter or materials discussed in the manuscript apart from those disclosed.

Writing assistance was utilized in the production of this manuscript. The authors would like to thank Marta Pulido, MD, and Content Ed Net for editing the manuscript and for editorial assistance; Rous de Castellar, $M D$, for protocol writing assistance; Marta Llorens, RN, and Ramón Dosantos, BSc, from Trial Form Support for protocol writing assistance and statistical analysis. 


\section{Key issues}

- Torasemide immediate release (IR) has a longer half-life and duration of action, and higher bioavailability compared with furosemide.

- Torasemide prolonged-release (PR) formulation has shown lower peak plasma levels while maintaining equivalent AUC of plasmatic levels compared with the IR formulation.

- PR and IR torasemide formulations were well tolerated and showed similar efficacy, tolerability and safety profiles in a population of mild-to-moderate hypertensive patients.

- Myocardial fibrosis is now considered a major determinant of altered diastolic filling and compromised systolic pump function leading to heart failure.

- Serum procollagen type I C-terminal propeptide (PICP), a biochemical marker of myocardial fibrosis, has been shown to be associated with the volume of myocardial tissue occupied by collagen fibers in hypertensive patients with and without heart failure.

- The TORAFIC study is a prospective, Phase IV, randomized, blinded end point, active-controlled drug clinical trial (PROBE design). Patients will be followed-up during the 8 months of the trial.

- The primary objective of this clinical trial is to determine the possible superiority of torasemide-PR compared with furosemide in reducing the serum concentration of PICP.

- This clinical trial will test whether torasemide-PR also possesses antifibrotic properties (as assessed by its ability to reduce serum PICP concentration) that, in turn, may provide an additional benefit beyond diuresis in patients with chronic congestive heart failure receiving this agent.

\section{References}

1 Mosterd A, Hoes AW, de Bruyne MC et al. Prevalence of heart failure and left ventricular dysfunction in the general population; The Rotterdam Study. Eur. Heart J. 20, 447-455 (1999).

2 Anguita Sánchez M, Crespo Leiro MG, de Teresa Galván E et al; PRICE study investigators. Prevalence of heart failure in the Spanish general population aged over 45 years. Rev. Esp. Cardiol. 61, 1041-1049 (2008).

3 McKee PA, Castelli WP, McNamara PM, Kannel WB. The natural history of congestive heart failure: the Framingham study. N. Engl. J. Med. 285, 1441-1446 (1971).

4 Cleland JG, Gemmell I, Khand A, Boddy A. Is the prognosis of heart failure improving? Eur. Heart J. 1, 229-241 (1999).

5 Cleland JG, Khand A, Clark A. The heart failure epidemic: exactly how big is it? Eur. Heart J. 22, 623-626 (2001).

6 Mann DL, Bristow MR. Mechanisms and models in heart failure: the biomechanical model and beyond. Circulation 111, 2837-2849 (2005).

7 Burlew BS, Weber KT. Connective tissue and the heart. Functional significance and regulatory mechanisms. Cardiol. Clin. 18, 435-442 (2000).

8 Chapman RE, Spinale FG. Extracellular protease activation and unraveling of the myocardial interstitium: critical steps toward clinical applications. Am. J. Physiol. Heart Circ. Physiol. 286, H1-H10 (2004).
9 Burlew BS, Weber KT. Cardiac fibrosis as a cause of diastolic dysfunction. Herz 27, 92-98 (2002).

10 Brower GL, Gardner JD, Forman MF et al. The relationship between myocardial extracellular matrix remodeling and ventricular function. Eur. J. Cardiothorac. Surg. 30, 604-610 (2006).

11 Díez J, González A, López B, Querejeta R. Mechanisms of disease: pathologic structural remodeling is more than adaptive hypertrophy in hypertensive heart disease. Nat. Clin. Pract. Cardiovasc. Med. 2, 209-216 (2005).

12 Querejeta R, Varo N, López B et al. Serum carboxy-terminal propeptide procollagen type $\mathrm{I}$ is a marker of myocardial fibrosis in hypertensive heart disease. Circulation 101, 1729-1735 (2000).

13 Querejeta R, López B, González A et al. Increased collagen type I synthesis in patients with heart failure of hypertensive origin: relation to myocardial fibrosis. Circulation 110, 1263-1268 (2004).

14 López B, Querejeta R, Varo N et al. Usefulness of serum carboxy-terminal propeptide of procollagen type I in assessment of the cardioreparative ability of antihypertensive treatment in hypertensive patients. Circulation 104, 286-291 (2001).

15 González A, López B, Ravassa S et al. Biochemical markers of myocardial remodelling in hypertensive heart disease. Cardiovasc. Res. 81, 509-518 (2009).

16 Swedberg K, Cleland J, Dargie H et al; Task Force for the Diagnosis and Treatment of Chronic Heart Failure of the European Society of Cardiology. Guidelines for the diagnosis and treatment of chronic heart failure: executive summary (update 2005): The Task Force for the Diagnosis and Treatment of Chronic Heart Failure of the European Society of Cardiology. Eur. Heart J. 26, 1115-1140 (2005).

17 Hunt SA, Abraham WT, Chin MH et al; American College of Cardiology; American Heart Association Task Force on Practice Guidelines; American College of Chest Physicians; International Society for Heart and Lung Transplantation; Heart Rhythm Society: ACC/AHA 2005 Guideline Update for the Diagnosis and Management of Chronic Heart Failure in the Adult: a report of the American College of Cardiology/American Heart Association Task Force on Practice Guidelines (Writing Committee to Update the 2001 Guidelines for the Evaluation and Management of Heart Failure): developed in collaboration with the American College of Chest Physicians and the International Society for Heart and Lung Transplantation: endorsed by the Heart Rhythm Society. Circulation 112, E154-E235 (2005).

18 Ishido H, Senzaki H. Torasemide for the treatment of heart failure. Cardiovasc. Hematol. Disord. Drug Targets 8, 127-132 (2008).

19 Cosín J, Díez J; TORIC investigators. Torasemide in chronic heart failure: results of the TORIC study. Eur. J. Heart Fail. 4, 507-513 (2002).

20 Dunn CJ, Fitton A, Brogden RN. Torasemide. An update of its pharmacological properties and therapeutic efficacy. Drugs 49, 121-142 (1995). 
21 Knauf H, Mutschler E. Clinical pharmacokinetics and pharmacodynamics of torasemide. Clin. Pharmacokinet. 34, 1-24 (1998).

22 Dodion L, Ambroes Y, Lameire N. A comparison of the pharmacokinetics and diuretic effects of two loop diuretics, torasemide and furosemide, in normal volunteers. Eur. J. Clin. Pharmacol. 31, 21-27 (1986).

23 Vargo DL, Kramer WG, Black PK, Smith WB, Serpas T, Brater DC. Bioavailability, pharmacokinetics, and pharmacodynamics of torsemide and furosemide in patients with congestive heart failure. Clin. Pharmacol. Ther. 57, 601-609 (1995).

24 Barbanoj MJ, Ballester MR, Antonijoan RM et al. A bioavailability/ bioequivalence and pharmacokinetic study of two oral doses of torasemide $(5 \mathrm{mg}$ and $10 \mathrm{mg}$ ): prolonged-release versus the conventional formulation. Clin. Exp. Pharmacol. Physiol. 36(5-6), 469-477 (2008) .

25 Gropper S, Rojas MJ, Guglietta A et al. Single and repeated-dose, randomized, cross-over, bioavailability clinical trial of torasemide immediate release compared to a new prolonged release formulation of torasemide. Basic Clin. Pharmacol. Toxicol. 99(Suppl. 1), 48 (2006) (Abstract).

26 Sutril Neo (torasemide prolonged release), summary of product characteristics. Ferrer Grupo, Barcelona, Spain (2006).

27 Roca-Cusachs A, Aracil-Vilar J, CalvoGómez $\mathrm{C}$ et al.; the Torasemide-PR in Hypertension Clinical Trial Investigators Group. Clinical effects of torasemide prolonged release in milt to moderate hypertension: a randomized noninferiority trial versus torasemide immediate release. Cardiovasc. Ther. 26, 91-100 (2008).

28 Veeraveedu PT, Watanabe K, Ma M et al. Comparative effects of torasemide and furosemide in rats with heart failure. Biochem. Pharmacol. 75, 649-659 (2008).
29 Veeraveedu PT, Watanabe K, Ma M et al. Torasemide, a long-acting loop diuretic, reduces the progression of myocarditis to dilated cardiomyopathy. Eur. J. Pharmacol. 581, 121-131 (2008).

30 López B, Querejeta R, González A, Sánchez E, Larman M, Díez J. Effects of loop diuretics on myocardial fibrosis and collagen type I turnover in chronic heart failure. J. Am. Coll. Cardiol. 43, 2028-2035 (2004).

31 López B, González A, Beaumont J, Querejeta $R$, Larman M, Díez J. Identification of a potential antifibrotic mechanism of torasemide in patients with chronic heart failure. J. Am. Coll. Cardiol. 50, 859-867 (2007).

32 López B, Querejeta R, González A, Beaumont J, Larman M, Díez J. Impact of treatment on myocardial lysyl oxidase expression and collagen cross-linking in patients with heart failure. Hypertension 53(2), 236-242 (2009).

33 Rector TS, Cohn JN. Assessment of patient outcome with the Minnesota Living with Heart Failure Questionnaire: reliability and validity during a randomized, double-blind, placebo-controlled trial of pimobendan. Am. Heart J. 124, 1017-1025 (1992).

34 Hansson L, Hedner T, Dahlöf B. Prospective randomized open blinded end-point (PROBE) study. A novel design for intervention trials. Prospective Randomized Open Blinded End-Point. Blood Press. 1, 113-119 (1992).

35 Paulus WJ, Tschöpe C, Sanderson JE et al. How to diagnose diastolic heart failure: a consensus statement of the diagnosis of heart failure with normal left ventricular ejection fraction by the Heart Failure and Echocardiography Associations of the European Society of Cardiology. Eur. Heart J. 28, 2539-2550 (2007).

36 European Society of Hypertension-European Society of Cardiology Guidelines Committee. 2003 European Society of Hypertension-European Society of Cardiology guidelines for the management of arterial hypertension. J. Hypertens. 21, 1011-1053 (2003).
37 Karch FE, Lasagna L. Toward the operational identification of adverse drug reactions. Clin. Pharmacol. Ther. 21, 247-254 (1977).

38 González A, López B, Díez J. New directions in the assessment and treatment of hypertensive heart disease. Curr. Opin. Nephrol. Hypertens. 14, 428-434 (2005).

\section{Affiliations}

- Javier Díez

Division of Cardiovascular Sciences,

Centre of Applied Medical Research, and

Department of Cardiology and

Cardiovascular Surgery, University Clinic,

Universidad de Navarra, Edificio CIMA,

Avda. Pío XII 55, E-31008, Pamplona,

Spain

Tel.: +349481947000

Fax: +34948 194716

jadimar@unav.es

- Antonio Coca

Hypertension Unit, Department of Internal Medicine, Institute of Medicine and Dermatology, Hospital Clinic (IDIBAPS), Universidad de Barcelona, Barcelona, Spain

- Eduardo de Teresa Department of Cardiology, Hospital Clínico, Málaga, Spain

- Manuel Anguita Department of Cardiology, Hospital Universitario Reina Sofía, Córdoba, Spain

- Alfonso Castro-Beiras

Department of Cardiology, Complejo Hospitalario Universitario de A Coruña, A Coruña, Spain

- Pedro Conthe Department of Internal Medicine, Hospital Gregorio Marañón, Madrid, Spain

- Erik Cobo Department of Statistics and Operational Research, Universidad Técnica de Cataluña, Barcelona, Spain

- Ester Fernández Medical Department, Novag (Ferrer Internacional), Barcelona, Spain 